\title{
A simple method for the formation of water-in-oil-in- water (W/O/W) double emulsions
}

Zhizhao Che ${ }^{1}$, Teck Neng Wong ${ }^{2}$, Nam-Trung Nguyen ${ }^{3}$

1. State Key Laboratory of Engines, Tianjin University, Tianjin, 300072, China.

2. School of Mechanical and Aerospace Engineering, Nanyang Technological University, 50 Nanyang Avenue, 639798, Singapore.

3. Queensland Micro- and Nanotechnology Centre, Griffith University, 170 Kessels Road, Brisbane QLD 4111, Australia.

\section{Abstract}

We proposed and experimentally demonstrated a simple, low-cost and reliable method for the formation of double emulsions. The formation process consists of two stages: (i) the formation of water-in-oil (W/O) droplets at a co-flowing structure formed by a syringe needle and a flexible microtubing, and (ii) the formation of water-in-oil-in-water $(\mathrm{W} / \mathrm{O} / \mathrm{W})$ compound droplets at the tip of the microtubing by buoyancy. Since the droplets flow directly from the first stage to the second stage in the tubing without any disturbance, common problems of other methods such as droplet coalescence, breakup, leakage or contamination can be avoided automatically. The breakup processes of the core droplets and the compound droplets are analyzed. The sizes of the core and shell droplets and the volume fraction in the compound droplets are controlled by adjusting the corresponding flow rates. Due to the simplicity of this method and the flexibility in controlling the properties of the double emulsions, this method shows great potential in the relevant applications.

\section{Introduction}

An emulsion is a mixture of a dispersed phase as droplets in another immiscible fluid that forms a continuous phase. Double emulsions are "emulsions within emulsions", i.e., droplets of one fluid are encapsulated inside droplets of another fluid. The shell fluid can serve as a barrier between the core droplets and the outer environment. 
Therefore, the core droplets can be loaded with many substances to achieve various functions, such as the encapsulation of pesticides, nutrients, drugs, and antibodies [1]. The compartmentalization of materials within droplets allows for a better control of the relevant process in various industrial applications of chemical engineering, material synthesis, biological analysis, pharmaceutical manufacturing, food engineering, etc $[2,3]$.

Conventional methods for making emulsions are typically form droplets in bulk, such as using shear generated by mechanical agitation. Therefore, the formation of individual droplets is poorly controlled and the product has a broad size distributions. Microfluidic devices offers an alternative to produce emulsions by making one droplets at a time [4-6]. Therefore, each droplet can be controlled precisely and the final product is highly monodisperse. This method can also produce double, triple or even higher order emulsions [7] by the series combination of several droplet generation units, such as the combination of two T-junctions in serial $[8,9]$ or two flow focusing geometries [10-12]. The sizes of the cores, the thickness of the shells, and the number of cores are controllable by their respective flow rates. Utada et al. $[13,14]$ designed a capillary device consisting a co-flowing geometry and a flow focusing geometry to form double emulsions. In the capillary device, two cylindrical capillaries were arranged end-to-end within a square capillary, and good coaxial alignment was achieved automatically by ensuring that the inner dimensions of the square capillary are the same as the outside diameters of the circular capillaries.

One major issue associated with the combination of droplet generation units is the wettability of the channels. Since the wettability determines the type of droplets to 
produce, such as water-in-oil droplets in hydrophobic channels or oil-in-water droplets in hydrophilic channels, the two units for the generation of double emulsions should have opposite wettability. The devices reported in the literature to generate double emulsions on microchips can be divided into two types according to whether the two units are fabricated on one or two chips [15]. In the two-chip design, the core droplets are firstly generated in one chip and then re-injected into another chip [16]. The wettability issue can be solved by using different materials with opposite wettability for the fabrication of the two chips. But this method involves the reinjection process, which may cause leakage or contamination. In contrast, the onechip design normally requires a precise modification of the surface wettability of one of the two units, which complicates the fabrication process [17-19].

Although a variety of the production methods for double emulsions have been proposed [20, 21], most of them requires complex fabrication or precise alignment. A simple and low-cost method is beneficial for practical applications. A simple and reliable method for the formation of double emulsions is proposed, which relies on easily available materials and a simple assembly procedure. The system consists of a co-flowing structure made of a plastic tubing and a syringe needle for the formation of core droplets, and the tubing tip in water to form compound droplets by buoyancy. The performance of this method was demonstrated by producing monodisperse compound droplets. The droplet size and the droplet distance were controlled by adjusting the respective flow rates. The reminder of this paper is organized as follows. The experimental details are described in Section 2, including the droplet formation device, the measurement system, and the fluid properties. The results are discussed in Section 3, including the formation of water-in-oil (W/O) droplets in the first step, and 
the formation of water-in-oil-in-water (W/O/W) compound droplets in the second step. Finally, conclusions are drawn in Section 4.

\section{Experiment}

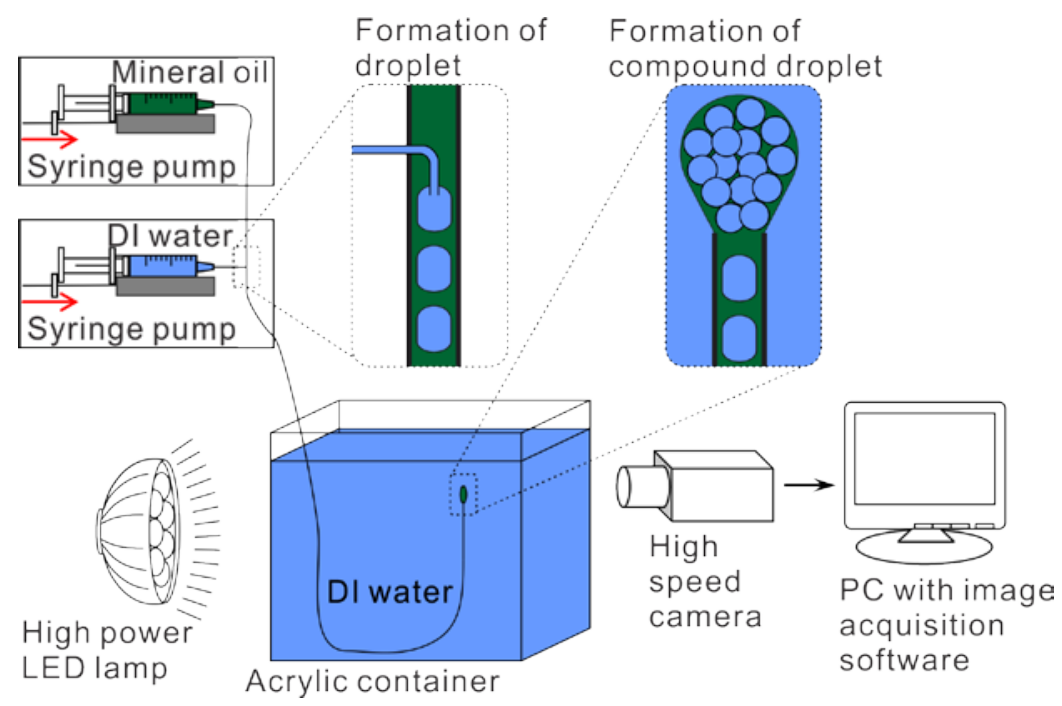

Figure 1 Schematic diagram of the experimental setup for the formation of double emulsions. W/O droplets are formed at a co-flowing structure consisting of a syringe tip and a plastic tubing. W/O/W compound droplets are formed at the tip of the tubing by buoyancy.

The experimental setup for the production of $\mathrm{W} / \mathrm{O} / \mathrm{W}$ double emulsions is shown in Figure 1. The formation process of the double emulsions consists of two steps: the first step is the formation of water droplets in oil at a co-flow structure, and the second step is the formation of compound droplets in water at a tip of microtubing. A blunt stainless syringe needle (Gauge 32 gauge with an inner diameter (ID) of 0.10 $\mathrm{mm}$ and an outer diameter (OD) of $0.24 \mathrm{~mm}$, EDF Singapore) was bent $90^{\circ}$ and pricked into a flexible tubing (ID $0.51 \mathrm{~mm}$, OD $1.52 \mathrm{~mm}$, Tygon microbore tubing, Cole Parmer) to form a co-flowing structure. Deionized (DI) water was introduced at the syringe needle, and mineral oil (Sigma M5904) at the tubing. The water and the oil streams met at the co-flowing structure to form water-in-oil droplets in the tubing. 
The two streams were introduced at constant flow rates into the system using glass syringes (Hamilton). Their flow rates were controlled separately by two syringe pumps (KD Scientific). A nonionic surfactant, Span 80 (Sigma S6760) was added into the mineral oil at a concentration of $2 \%$ by weight to assist the formation of droplets and to stabilize them against coalescence. The tubing with the co-flowing structure was clipped to a glass slide and the formation process of water-in-oil droplets was observed on a microscope (Nikon Eclipse TE2000-S) and recorded using a high speed camera (Phantom M310).

To form compound droplets at the tip of the flexible tubing, the tip of the tubing was immersed in DI water in a rectangular acrylic container. The tubing tip was clipped upward in DI water and was adjusted by an xyz linear translation stage. The water-inoil droplets, after the formation at the co-flowing structure, leave the tubing and form water-in-oil-in water compound droplets at the tip of the tubing. A high-speed camera (Phantom M310) with a macro lens (Nikon Micro-Nikkor 60mm f/2.8D) was placed horizontally to film the process of the growth and breakup of compound droplets. A high power LED lamp (GSvitec MultiLED, Germany) with a light diffuser was used to backlit the compound droplets for high-speed imaging. The images were processed using a customised MATLAB program.

The viscosity and the density of the mineral oil with the surfactant are $0.0238 \mathrm{~Pa} \cdot \mathrm{s}$ and $0.88 \times 10^{-3} \mathrm{~kg} / \mathrm{m}^{3}$, respectively. The dynamic interfacial tension against DI (measured using the pendant drop method on an FTA200 system, First Ten Ångstroms, USA) is shown in Figure 2, and the static value is 3.65 mN/s. 


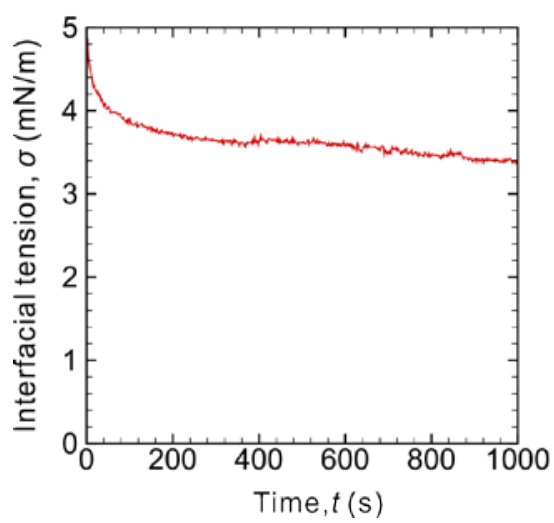

Figure 2 Dynamic interfacial tension of mineral oil with surfactant Span 80 at a concentration of $2 \%$ by weight in DI water at $25^{\circ} \mathrm{C}$.

\section{Results and discussion}

\subsection{Formation of water-in-oil droplets}

\subsubsection{Process of droplet formation}

To ensure the monodispersity of the water-in-oil droplets formed in the first step, the droplet formation process at the co-flowing structure was maintained in the dripping and squeezing regimes by setting the flow rates of the water and oil phases. Figure 3 shows the droplet formation process at the co-flowing structure with a typical set of flow rates. The formation of a droplet in the tubing has three stages. After the breakup of the previous droplet, a small finger of water forms at the tip of the needle (Figure 3a). The size of the water finger grows as more water is supplied. When the size of the finger head becomes significantly larger than the outer diameter of the syringe needle, the flow of the continuous phase (oil) is partially blocked by the water finger. As the water finger grows further, it occupies most of the entire cross section of the tubing, and is deformed by the flow of the continuous phase as shown in Figure 3e.

The water finger then is squeezed by the continuous phase, and the neck connecting the water finger and the upstream fluid begins to shrink due to the pressure and 
viscous stress applied on the interface by the continuous phase (Figure 3g). The shrinkage of the neck continues as the pressure and the viscous stress build up until the diameter of the neck is below a threshold. The surface tension force, being proportional to the curvature of the interface, dominates in the process, and accelerates the pinch-off of the neck until the breakup (Figure 3l).

After a water droplet forms by the breakup of the water finger, the droplet gradually restores its plug shapes moving to the downstream and a small spherical water finger forms again at the tip of the syringe needle, Figure $3(\mathrm{~m}-\mathrm{o})$. This process of growth, pinch-off, and restoration repeats to form droplets periodically as more liquid are supplied from the upstream.

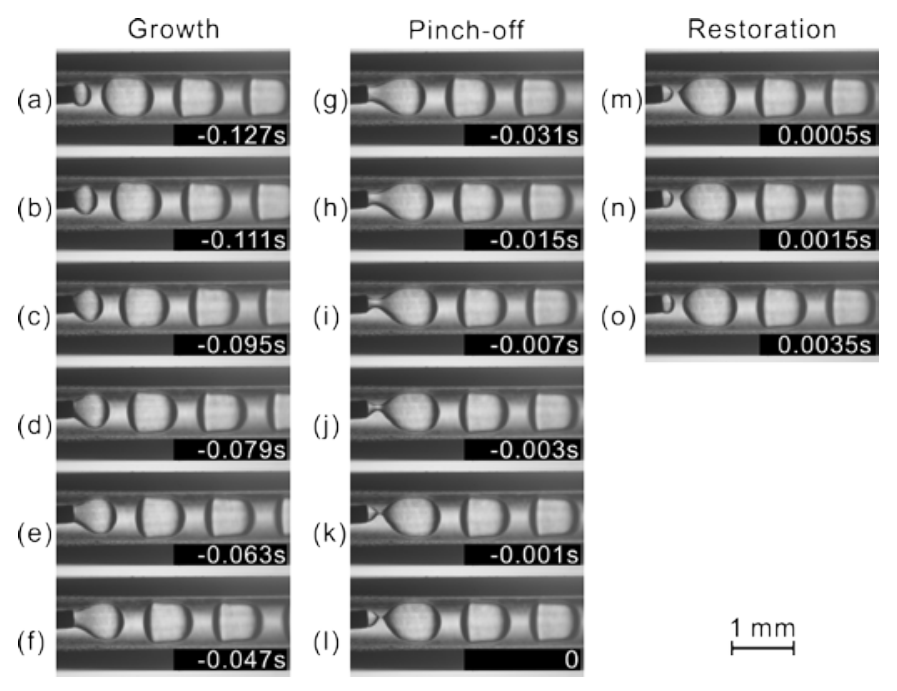

Figure 3 Droplet formation at the co-flowing structure in the tubing at a typical set of the flow rates of water and oil phases $\left(Q_{\mathrm{w}}=Q_{\mathrm{o}}=3 \mathrm{ml} / \mathrm{hr}\right)$. The images are not to scale in the spanwise direction due to the optical refraction at the tubing surface.

\subsubsection{Effect of flow rate of the oil phase}

To study the effect of the flow rate of the oil (shell) phase on the droplet formation process, the oil flow rate $Q_{\mathrm{o}}$ was varied while the water (core) flow rate $Q_{\mathrm{w}}$ was fixed. 
Figure 4a-h show the snapshots at the instance of breakup. Figure $4 \mathrm{i}$ plots the corresponding droplet sizes and the droplet distances in the tubing versus the oil flow rate. Increasing the flow rate of the oil phase decreases the size of the droplets and increases the distance between the formed droplets in the tubing. At a higher flow rate, the oil phase applies a higher shear force on the surface of the liquid finger. The higher shear shortened the growth process and accelerated the pinch-off of droplets. This result demonstrates the capability of our method in obtaining droplets of different sizes and the ease in controlling the droplet size by adjusting the flow rates of the core and the shell phases.
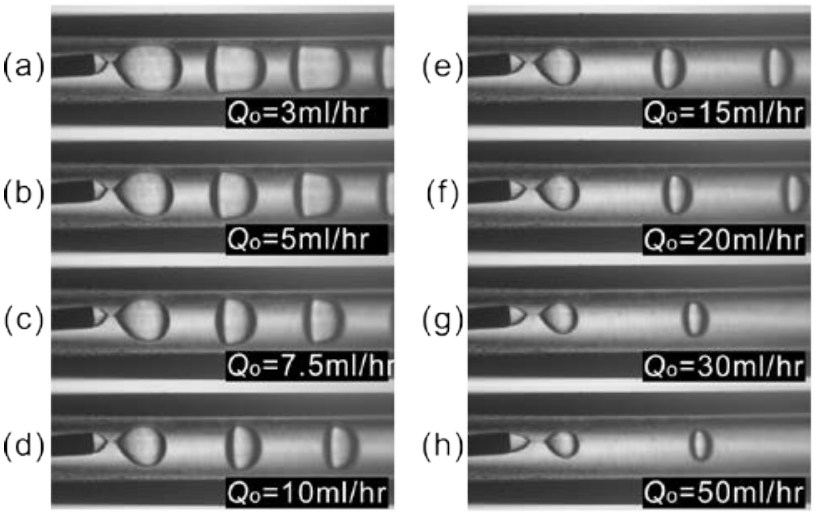

$1 \mathrm{~mm}$

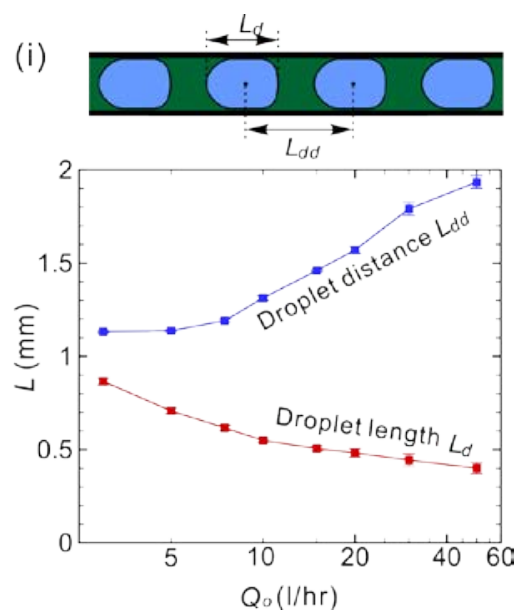

Figure 4 Droplet formation at the co-flowing structure in a tubing at different flow rates of the oil phase. The flow rates of oil $Q_{0}$ are provided in the figures, while the flow rate of water is fixed at $Q_{\mathrm{w}}=3 \mathrm{ml} / \mathrm{hr}$. (a-h) Snapshots at the instance of breakup. The images are not to scale in the spanwise direction due to the optical refraction at the tubing surface. (i) Droplet length and droplet distance in the tubing. The droplet length was measured from the nose to the tail of the droplet, while the droplet distance was measured from the centres of the droplets in the images.

\subsubsection{Effect of the water-oil total flow rate}

To study the effect of the total flow rate of the oil phase $Q_{0}$ and the water phase $Q_{\text {w }}$ on the droplet formation process, the total flow rate was increased gradually with 
the flow rate ratio fixed to 1 . The snapshots at the instance of breakup are shown in Figure 5a-j and the corresponding sizes of droplets are shown in Figure 5k. The droplet size and the droplet distance almost do not vary with the total flow rates. As the flow rate of the oil phase increases, the oil phase can apply a larger stress on the water finger and shorten the time required to formed a droplet, but the flow of the droplet phase also increases proportionally to introduce the almost the same amount of fluid into the water finger. Therefore, at a fixed flow rate ratio, the total flow rate could not significantly affect the droplet size and the droplet distance. This result indicates that the system can work in a wide range of parameters without affecting the quality of the droplets.


Figure 5 Droplet formation at the co-flowing structure in the tubing at different total flow rates with a fixed flow rate ratio $Q_{0} / Q_{\mathrm{w}}=1$. (a-j) Snapshots at the instance of droplet breakup. The images are not to scale in the spanwise direction due to the optical refraction at the tubing surface. (k) Droplet length and droplet distance in the tubing. 


\subsection{Formation of W/O/W compound droplets}

\subsubsection{Formation dynamics of compound droplets}
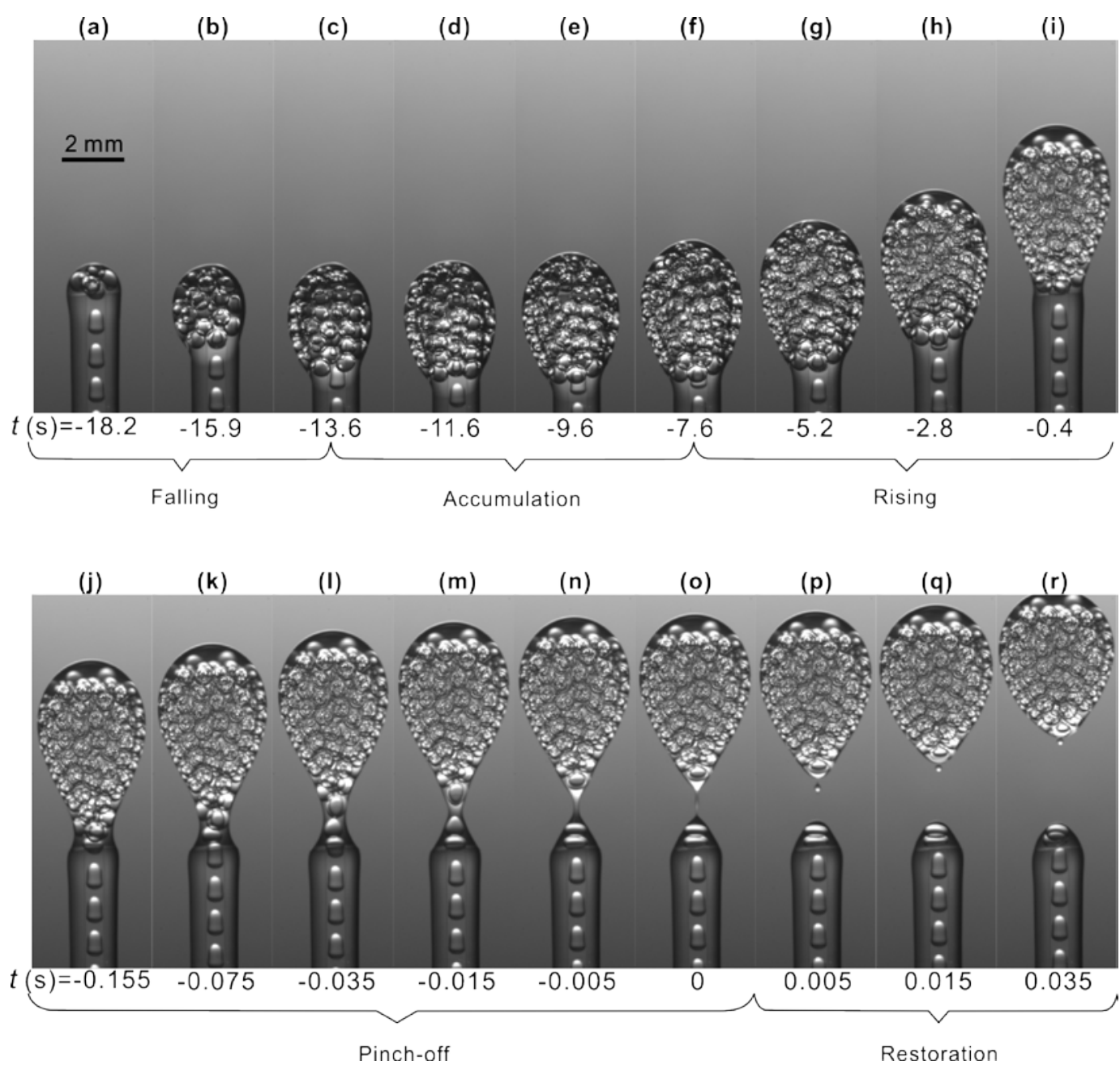

Figure 6 Process of the formation of W/O/W compound droplets at a setting of flow rates. The flow rates of water and oil are $3 \mathrm{ml} / \mathrm{hr}$ and $3 \mathrm{ml} / \mathrm{hr}$, respectively. The formation process of a compound droplet consists of five stages: (1) receding of the contact line, (2) accumulation of liquids at the liquid finger, (3) rising of the contact line, (4) pinch-off of the neck of the finger, and (5) restoration of the interface shapes. A video for this process is available as Supplementary Material.

Following the formation of water-in-oil droplets at the co-flowing structure in the tubing, the droplet train flows downstream to the tip of the tubing to form $\mathrm{W} / \mathrm{O} / \mathrm{W}$ compound droplets. Since the droplets flow directly from the co-flowing structure to 
the tubing tip without any disturbance or fittings and the tubing used can be very long, the droplets can be transported without any coalescence or breakup, and leakage or contamination can be automatically avoided as well. Figure 6 shows the typical process of the formation of $\mathrm{W} / \mathrm{O} / \mathrm{W}$ compound droplets by buoyancy. After the breakup of the previous compound droplet, a small compound liquid finger forms at the tip of the tubing, Figure 6a. As more liquid is supplied to the tip, the contact line of the liquid falls, this is because the wettability of the oil with surfactant is higher than that of DI water. The movement of the contact line is accompanied by the shape variation of the liquid finger, which can be quantified by the position of the apex of the liquid finger (Figure 7a). In this stage, the shape of the liquid finger is mainly determined by the surface tension force. As more liquid is supplied to the tip, the size of the liquid finger increases, and the gravitational force increases. At this stage, the surface tension force and the gravitation force are at the same scale. Therefore, the liquid finger bulges but does not move significantly during the accumulation stage, Figure 6c-f. As more liquid is supplied to the tip, the gravitational force becomes stronger than the surface tension force. Therefore, the liquid finger rises and the contact line on the tubing also rises, Figure 6g-i. When the contact line rises to the tip of the tubing, the gravitational force cannot be balanced by the surface tension force. Thus, the neck of the compound liquid finger starts to shrink, which indicates the end of the rising stage and the beginning of the pinch-off stage, Figure 6i.

The pinch-off stage in characterised by the thinning of the liquid thread connecting the compound droplet and the upstream liquid. This stage accelerated as the thread became thinner (see Figure 7b), because the surface tension force, as the dominant force of the pinch-off process, is proportional to the curvature of the interface. The 
pinch-off stage ends as the droplet detaches from the tip of the tubing, Figure 6o. After the detachment, the compound droplets tend to restore the spherical shape because of the surface tension. At the tip of the tubing, the liquid finger also immediately restores to the shape of a spherical cap, Figure 6p. During the pinch-off process, a small satellite droplet forms at the liquid thread. Due to the restoration of the compound droplets, the satellite droplet flow upward rapidly due to the upward flow induced by the surface tension of the compound droplet, Figure 6q.


Figure 7 (a) Evolution of the position of the apex of the liquid finger during the formation of the compound droplet. The position is relative to the tip of the tubing. The sudden decrease of the apex position corresponds to the breakup of the compound droplet. (b) Evolution of the thread diameter during the pinch-off of the compound droplet. 


\subsubsection{Effect of flow rate ratio}
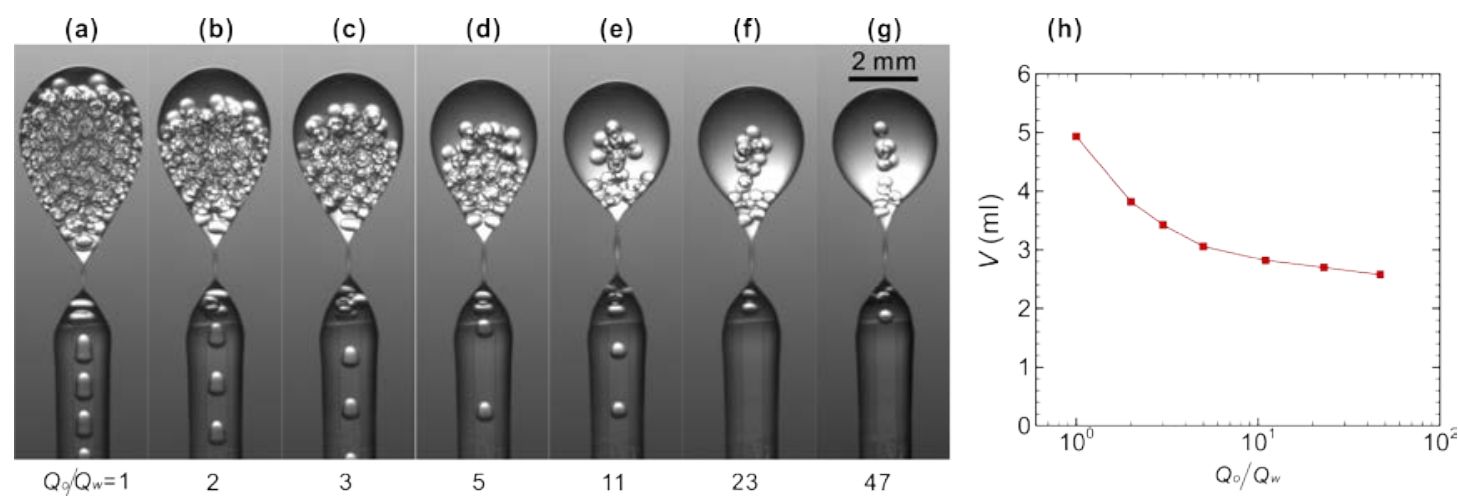

Figure 8 Formation of compound droplets at different flow rate ratios. The total flow rate of oil and water are fixed at $Q_{\mathrm{o}}+Q_{\mathrm{w}}=6 \mathrm{ml} / \mathrm{hr}$. (a-g) Snapshots at the instance of droplet breakup. (h) Volume of compound droplets $V$.

The effect of the oil-water flow rate ratio on the formation process was studied by increasing the flow rate of oil $Q_{0}$ with a fixed the total flow rate of water and oil at the co-flowing structure. The droplet shape at the instant of the breakup are shown in Figure 8(a-g), and the corresponding size of the compound droplets is plotted against the flow rate ratio in Figure 8(h). Increasing oil-water flow rate ratio at the co-flowing droplet generation unit decreases the size of the core water droplets. The distance between two droplets also increases, as discussed in Section 3.1. The volume fraction of water in the compound droplets decreases significantly as it is defined by the flow rate ratio. In addition, because the oil phase has a much lower density than water, the gravitational force becomes more significant at high flow rate ratios. Therefore, the size of the compound droplets becomes smaller.

\section{Conclusions}

The present paper reports a simple method to form double emulsions, The poof of concept was demonstrated with the formation of water-in-oil-in-water compound droplets. The formation process consists two stages. In the first stage, water-in-oil 
droplets were produced at a co-flowing structure formed by a syringe needle and a flexible microtubing. In the second stage, water-in-oil-in-water compound droplets were produced at the tip of the microtubing by buoyancy. Since the droplets flow directly from the first stage to the second stage in the tubing without any disturbance or fittings, the droplets can be transported without any coalescence or breakup. Leakage or contamination are automatically avoided as well. The formation processes for droplets and compound droplets were analyzed. The effects of the flow rates of the continuous and the dispersed phases and their ratio were studied. The results showed that the size of the inner droplets and the volume fraction of the compound droplets could be controlled by adjusting the flow rates of the two phases. Since the first stage of the formation is in the squeezing and the dripping regimes and the second stage is controlled by gravity, the process can be achieved with high repeatability and warrants the monodispersity of the compound droplets. Due to the simplicity of this method and the flexibility in controlling the size of the core droplets and the volume fraction, this method has great potential applications in a wide range of areas, such as chemical engineering, particle synthesis, biological analysis, pharmaceutical manufacturing, and food engineering.

\section{References}

[1] W. Wang, M.-J. Zhang, L.-Y. Chu, Microfluidic approach for encapsulation via double emulsions, Current opinion in pharmacology, 18 (2014) 35-41.

[2] S.Y. Teh, R. Lin, L.H. Hung, A.P. Lee, Droplet microfluidics, Lab Chip, 8(2) (2008) 198-220.

[3] S.S. Datta, A. Abbaspourrad, E. Amstad, J. Fan, S.H. Kim, M. Romanowsky, H.C. Shum, B. Sun, A.S. Utada, M. Windbergs, 25th anniversary article: Double emulsion templated solid microcapsules: Mechanics and controlled release, Adv Mater, 26(14) (2014) 2205-2218.

[4] T. Thorsen, R.W. Roberts, F.H. Arnold, S.R. Quake, Dynamic pattern formation in a vesicle-generating microfluidic device, Phys Rev Lett, 86(18) (2001) 4163-4166.

[5] S.L. Anna, N. Bontoux, H.A. Stone, Formation of dispersions using "flow focusing" in microchannels, Appl Phys Lett, 82(3) (2003) 364-366. 
[6] G.F. Christopher, S.L. Anna, Microfluidic methods for generating continuous droplet streams, J Phys D Appl Phys, 40(19) (2007) R319-R336.

[7] A. Abate, D. Weitz, High - order multiple emulsions formed in poly (dimethylsiloxane) microfluidics, Small, 5(18) (2009) 2030-2032.

[8] T. Nisisako, S. Okushima, T. Torii, Controlled formulation of monodisperse double emulsions in a multiple-phase microfluidic system, Soft Matter, 1(1) (2005) 23-27.

[9] S. Okushima, T. Nisisako, T. Torii, T. Higuchi, Controlled production of monodisperse double emulsions by two-step droplet breakup in microfluidic devices, Langmuir, 20(23) (2004) 9905-9908.

[10] A.R. Abate, D.A. Weitz, High-order multiple emulsions formed in poly(dimethylsiloxane) microfluidics, Small, 5(18) (2009) 2030-2032.

[11] J. Wan, A. Bick, M. Sullivan, H.A. Stone, Controllable microfluidic production of microbubbles in water-in-oil emulsions and the formation of porous microparticles, Adv Mater, 20(17) (2008) 3314-3318.

[12] N.-N. Deng, Z.-J. Meng, R. Xie, X.-J. Ju, C.-L. Mou, W. Wang, L.-Y. Chu, Simple and cheap microfluidic devices for the preparation of monodisperse emulsions, Lab Chip, 11(23) (2011) 3963-3969.

[13] A.S. Utada, E. Lorenceau, D.R. Link, P.D. Kaplan, H.A. Stone, D.A. Weitz, Monodisperse double emulsions generated from a microcapillary device, Science, 308(5721) (2005) 537-541.

[14] E. Lorenceau, A.S. Utada, D.R. Link, G. Cristobal, M. Joanicot, D.A. Weitz, Generation of polymerosomes from double-emulsions, Langmuir, 21(20) (2005) 9183-9186.

[15] T. Nisisako, Microstructured devices for preparing controlled multiple emulsions, Chem Eng Technol, 31(8) (2008) 1091-1098.

[16] I. Batinic-Haberle, A. Tovmasyan, E.R. Roberts, Z. Vujaskovic, K.W. Leong, I. Spasojevic, SOD therapeutics: Latest insights into their structure-activity relationships and impact on the cellular redox-based signaling pathways, Antioxidants \& redox signaling, 20(15) (2014) 2372-2415.

[17] A.R. Abate, J. Thiele, M. Weinhart, D.A. Weitz, Patterning microfluidic device wettability using flow confinement, Lab Chip, 10(14) (2010) 1774.

[18] V. Barbier, M. Tatoulian, H. Li, F. Arefi-Khonsari, A. Ajdari, P. Tabeling, Stable modification of PDMS surface properties by plasma polymerization: Application to the formation of double emulsions in microfluidic systems, Langmuir, 22(12) (2006) 5230-5232.

[19] A.R. Abate, J. Thiele, D.A. Weitz, One-step formation of multiple emulsions in microfluidics, Lab Chip, 11(2) (2011) 253.

[20] D. Saeki, S. Sugiura, T. Kanamori, S. Sato, S. Ichikawa, Microfluidic preparation of water-in-oil-in-water emulsions with an ultra-thin oil phase layer, Lab Chip, 10(3) (2010) 357-362.

[21] F.-C. Chang, Y.-C. Su, Controlled double emulsification utilizing 3D PDMS microchannels, J Micromech Microeng, 18(6) (2008) 065018. 